\title{
Folded metaporous material for sub-wavelength and broadband perfect sound absorption
}

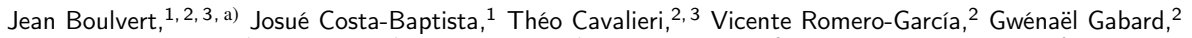

Edith Roland Fotsing, ${ }^{1}$ Annie Ross, ${ }^{1}$ Maxime Perna, ${ }_{1}^{1}$ Jacky Mardjono, ${ }^{3}$ and Jean-Philippe Groby ${ }^{2}$

${ }^{1)}$ Laboratoire d'Analyse Vibratoire et Acoustique, LAVA, Mechanical engineering, Polytechnique Montréal, H3T 1J4, Montréal,

Québec, Canada

${ }^{2)}$ Laboratoire d'Acoustique de l'Université du Mans, LAUM - UMR CNRS 6613, Le Mans Université, Avenue Olivier Messiaen,

72085 Le Mans Cedex 9, France

${ }^{3)}$ Safran Aircraft Engines, Rond Point René Ravaud - Réau, 77550 Moisy-Cramayel Cedex France

(Dated: 10 December 2020)

This letter reports a folded metaporous surface optimized to achieve subwavelength and broadband perfect absorption. Their unit cell is composed of four different helicoidal cavities filled by porous media which are structured and quasiisotropic micro-lattices with variable lattice constant. The effective thickness and intrinsic losses of each helicoidal cavity can be adjusted independently by varying their macro and micro-structures, namely the number of revolution of the folded structure and the lattice constant of the micro-lattice. An analytical model predicting the physical properties of this metaporous surface is developed. The macro and micro-structures are then jointly optimized for sub-wavelength broadband perfect absorption. Finally, the system is $3 \mathrm{D}$ printed and experimentally tested. The experimental results are found in good agreement with the theory and show an almost perfect absorption over a frequency range out of reach for the homogeneous constitutive porous medium and the only helicoidal cavities.

In many practical applications, acoustic treatments are constrained to be thin to limit their associated bulk and mass. The downside is that it conflicts with the broadband absorption/attenuation of low and medium-frequency noise. Indeed, the first resonance frequency $f_{0}$ of most acoustic treatments relying on Helmholtz (HR) or quarter-wavelength resonators (QWR) such as liners is inversely related to their effective acoustic thickness $L_{a}$, i.e., the effective distance over which the pressure wave propagates. For example, $f_{0}$ of a $L_{a}$-thick straight QWR corresponds to a wavelength $\lambda=4 L_{a}$ when no losses are accounted for.

Open-cell porous layers form another class of widely used acoustic treatments. Their ability to operate as broadband acoustic absorbers is well established. However, their low and medium-frequency efficiency is limited by their thickness and their intrinsic loss mechanisms ${ }^{1}$. As the sound speed is smaller in porous materials than in air, the so-called "quarterwavelength resonance" of such layer is slightly shifted towards lower frequencies in comparison to that of an airfilled QWR and $\lambda \approx 5 L_{a}$ is usually observed for a fibrous layer $^{2}$. Open-cell porous materials can be $3 \mathrm{D}$ printed allowing the control of their microstructure and the tunning of and their macroscopic properties ${ }^{3-6}$. An efficient way to tackle the absorption of wavelengths many times larger than the thickness of the treatment consists in folding its constitutive elements ${ }^{7-9}$. The equality between the effective acoustic thickness $L_{a}$ and the bulk treatment thickness $L_{b}$ is lifted and $L_{a} \geq L_{b}$, shifting down the corresponding resonance frequency while maintaining the treatment thickness. First experimental and theoretical explorations were carried out on folded aeronautic liners ${ }^{10,11}$. Models and geometries are still under development ${ }^{7,8}$. More recently, the folding concept

a) Author to whom correspondence should be addressed: jean.boulvert@univ-lemans.fr has been revisited to design notably acoustic metamaterial absorbers in the low-frequency range ${ }^{9}$. Folding the geometry of the acoustic treatment elements helps in increasing the density of states at low frequencies. However, perfect absorption requires in addition to match the impedance of the acoustic treatment with that of the surrounding medium. The energy leakage of the acoustic treatment to the surrounding medium must be perfectly balanced by its inherent losses to satisfy this condition, also known as critical coupling condition ${ }^{12}$. Recently the concepts of folding and critical coupling have been combined to design sub-wavelength perfect absorbers, based on folded QWR ${ }^{13}, \mathrm{HR}^{14}$, or other type of resonators ${ }^{15}$. However, the absorption bandwidths of this kind of perfect absorbers are narrow due to the high quality factor of their resonances. Therefore combining a collection of detuned elements can lead to broadband perfect absorption ${ }^{16}$.

The concept of metaporous surface ${ }^{17}$ relies on the embedding of resonant elements ${ }^{18-21}$ to enhance the density of state at low-frequency of a porous layer to achieve broadband and low-frequency absorption. The low-frequency absorption of the metaporous surface is higher than that of the single porous layer. The opposite approach, followed in this letter, consists in using properly designed porous materials to enhance the absorption of folded metamaterials

In this work, we theoretically and experimentally report folded resonators filled with 3D printed open-cell porous materials, the resulting low quality factor resonances of which being critically coupled at normal incidence. Four different folded porous media (FPM) working at different frequencies are designed and assembled in a metaporous surface (MpS) presenting a broadband quasi-perfect absorption at normal incidence for $5.6 L_{b}<\lambda<9.9 L_{b}$. The MpS is mounted in a square waveguide of width $W=41.9 \mathrm{~mm}$ with a rigid backing and excited by a normal incident plane wave to mimick an infinite periodic acoustic treatment ${ }^{22}$. This configuration is analytically modeled by the transfer matrix method inside the 

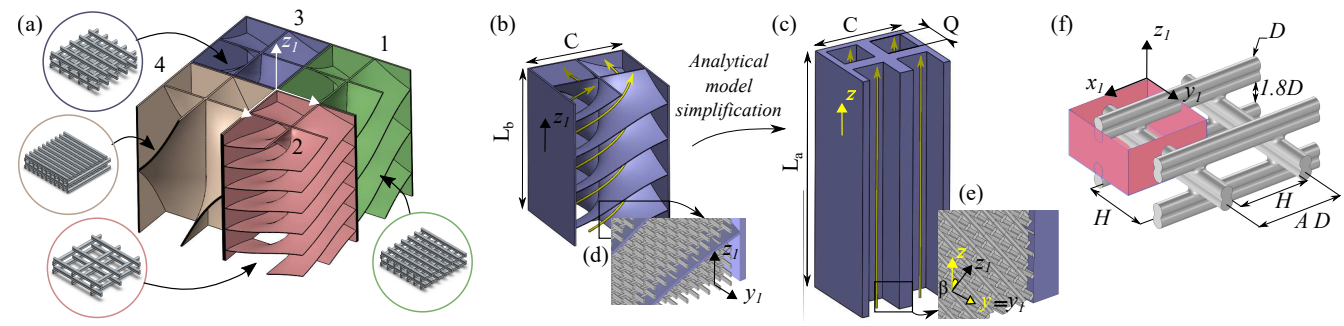

FIG. 1. Diagram of the MpS. (a) Schematic of parallel assembly of four FPMs of different $\xi$ filled by MLs of different $I F$. (b) Single FPM, $\xi=1$, impervious structure. The gold arrows represent the circular helix of mean acoustic path of length $L_{a}$. (c) Impervious structure of a FPM analytical model simplification. (d) FPM filled by the ML, detail view. (e) FPM analytical model simplification detail view. (f) ML idealized micro-structure. The box delimits a unit cell.

FPM coupled with a Bloch wave decomposition outside the $\mathrm{MpS}$ in order to account for the possible coupling between the FPM. This analytical model is used to calculate the reflection coefficient of the MpS. The MpS geometry is then optimized to maximize its absorption coefficient over a broadband frequency range by critically coupling each FPM. The optimized $\mathrm{MpS}$ is 3D printed and experimentally tested. Experimental results are in good agreement with both the analytical result and by Finite Element Method (FEM) numerical simulations.

A schematic of MpS is shown in Fig. 1(a). It consists of four impervious folded helicoidal structures, inspired from Refs. [23 and 24], filled with different porous materials. The FPMs are labeled as shown in the Figs. 1(a) and 2(b)(c), their relative position is important. To create a FPM, a Greek cross of width $C / \sqrt{2}$ is run along the vertical $\left(O, \mathbf{z}_{\mathbf{1}}\right)$ axis over an helix, generating a 4 branch helicoid. The later is then encapsulated in a square cuboid of height $L_{b}$ and width $C=W / 2$, see Fig. 1(b). Other folding strategies could be applied. The main advantage of this strategy is that the folding of each FPM can be done independently of that of the other FPMs: the FPMs are not tangled together. The number of revolutions operated by the helix over the height $L_{b}$ is denoted $\xi$. The generated helicoidal cavities are then filled with a homogeneous anisotropic porous material as depicted in Fig. 1(d) creating the FPM. The pressure waves propagate along an helical mean path depicted by the gold arrows in Fig. 1(b) with a length $L_{a} \geq L_{b}$.

The considered filling porous material is the micro-lattice (ML) depicted in Fig. 1(f) composed of a superposition of parallel fibers orthogonally alternating in the $\left(O, \mathbf{y}_{1}, \mathbf{z}_{1}\right)$ plane Each fiber is a combination of two filaments of diameter $D=200 \mu \mathrm{m}$ merged with one another in the $\left(O, \mathbf{z}_{\mathbf{1}}\right)$ direction. The distance between two micro-layers in the $\left(O, \mathbf{z}_{1}\right)$ direction is $0.9 \mathrm{D}$ to guarantee the bonding between filaments. Then, the representative unit-cell consists in the junction of two orthogonal fibers as highlighted in Fig. 1(f). It is described by two micro-structural parameters: the filament diameter $D$ and the spacing between two adjacent fibers in the $\left(O, \mathbf{x}_{\mathbf{1}}, \mathbf{y}_{\mathbf{1}}\right)$ plane, i.e., the lattice constant $A D$ where $A>1$. The pore size is defined here as the minimum distance between two adjacent fibers. In the $\mathbf{z}_{\mathbf{1}}$ direction, the pores are square and the pore
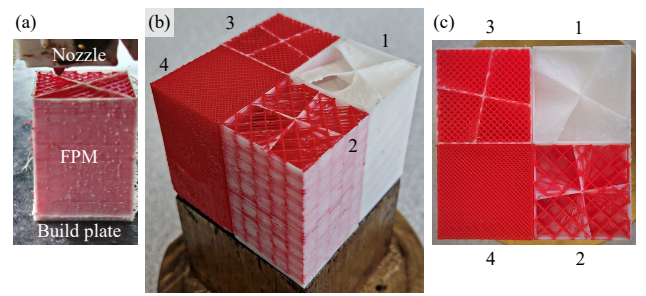

FIG. 2. Pictures of optimized and 3D printed MpS. (a) Single FPM being 3D printed. (b) Lateral and (c) top view of MpS.

size is equal to $H=D(A-1)$. In the $\mathbf{x}_{\mathbf{1}}$ and $\mathbf{y}_{\mathbf{1}}$ directions, the pores are rectangular and of size $H \times 1.8 D$. Thus, the medium is structured, periodic and quasi-isotropic. The manufacturing variable that regulates the lattice constant is the "infill factor" $I F$, which can only take integer percent values. The relation between the infill factor $I F$ and lattice constant is simply $I F(\%)=100 / A$.

The FPM are made by the Fused Deposition Modeling (FDM) additive manufacturing technique: the printer successively adds micro-layers made of extruded filaments on top of each other. The extruded material is polylactic acid (PLA). A $400 \mu \mathrm{m}$ diameter nozzle manufactures the impervious structure and a $200 \mu \mathrm{m}$ diameter manufactures the ML. A $1 \mathrm{~mm}$ thick solid layer is added at the bottom of the FPM to guarantee that they are rigidly backed. The four FPMs are printed separately and then assembled with vacuum grease on their external walls. Pictures are shown in Fig. 2 illustrating the manufacturing of a FPM (a) and the assembled MpS (b). Supp. mat. gives more information about the manufacturing process.

The skeleton of the porous medium, i.e., the ML is rigid enough to be assumed motionless. The porous medium can thus be modeled as an equivalent fluid ${ }^{1}$. It accounts for viscous and thermal losses occurring inside the porous medium through its equivalent complex and frequency dependent density $\boldsymbol{\rho}$ and bulk modulus $K$. An equivalent quasi-isotropic fluid implies that $K$ is scalar while $\boldsymbol{\rho}$ is a $3 \times 3$ diagonal ma- 
trix in the $\left(O, \mathbf{x}_{1}, \mathbf{y}_{1}, \mathbf{z}_{1}\right)$ frame where $\rho_{x_{1} x_{1}}=\rho_{y_{1} y_{1}} \neq \rho_{z_{1} z_{1}}$. According to the well-known Johnson-Champoux-AllardLafarge (JCAL) model ${ }^{25}$, the expression of the equivalent fluid properties can be approximated by means of six parameters related to the porous medium micro-structure in the case of isotropic media ${ }^{25}$ and nine parameters in the case of the considered quasi-isotropic medium ${ }^{26}$ (see supp. mat.).

The lattice constant, governed by the $I F$ in practice, directly influences the ML micro-structure and consequently its equivalent fluid properties. In particular, reducing the lattice constant, i.e., stepping the $I F$ up, increases the intrinsic losses of the ML. The JCAL parameters dependence on the IF of the ML were obtained by a two scales asymptotic homogenization method ${ }^{27-29}$ combined to the inverse characterization $^{30}$ of a set of eight homogeneous samples followed by an interpolation over the $I F$ range $^{6}$. The JCAL parameters of the characterized samples along with their interpolation $(I F \in[3 ; 60] \%)$ are presented in the supp. mat.

The MpS will be placed in a rigid backing configuration inside a square cross section waveguide and excited by a normal incidence plane wave. The walls of the waveguide act as mirrors for the pressure waves, a periodic pattern of MpS is then virtually created. Therefore, an infinite wall covered by a periodic distribution of the $\mathrm{MpS}$ will be evaluated. This configuration is first theoretically and numerically studied.

The analytical model does not account for the complex geometry of the FPM. Instead, each FPM of height $L_{b}$, and of cross sectional surface $C^{2}$ is approximated as a quadruplet of straight QWR filled by porous media. They are of normal axis $(O, \mathbf{z})$, of thickness $L_{a}(\xi) \geq L_{b}$ and of square cross section $Q^{2}(\xi) \leq C^{2} / 4$ (see Fig. 1(c). For geometrical reasons and because the ML is quasi-isotropic, the principal direction $\mathbf{z}_{1}$ of the ML filling the QWR forms an angle $\beta$ with $\mathbf{z}$ around $(O, \mathbf{y})=\left(O, \mathbf{y}_{\mathbf{1}}\right)$ (see Fig. 1(e) in the analytical model. The latter uses a transfer matrix formulation adapted to anisotropic media $^{31}$ for the propagation in the porous-filled QWR and a modal decomposition method considering Bloch modes in the surrounding air. Bloch waves account for the possible evanescent coupling between the different FPM in the MpS. From the numerical point of view a full wave numerical simulation by FEM with the real geometry is used. Further information is given in the supp. mat.

In both models, the MLs are represented by quasi-isotropic equivalent fluids defined by the JCAL model with the parameters obtained from inverse characterization. The walls of the structures are perfectly impervious and smooth and their induced losses are not accounted for $^{32}$. Validation tests have shown, on the one hand, that the analytical model leads to very accurate predictions of the simplified geometry. On the other hand, the simplification of a FPM by a quadruplet of QWR leads to slight shifts of the absorption coefficient in comparison to FEM predictions that grows with frequency.

The analytical model is used to optimize the geometry of a $L_{b}=30 \mathrm{~mm}$ thick $\mathrm{MpS}$ to produce perfect absorption over a broadband frequency range. The optimization procedure, based on an iterative Nelder-Mead algorithm, aims to maximize the absorption coefficient in a targeted range of frequencies. To do so, the optimal folding of each FPM, governed by their $\xi$, and their associated ML, governed by their $I F$ are optimized. During the optimization, and for manufacturing reasons, $I F$ is restricted to integer values comprised between $3 \%$ and $60 \%$. $\xi$ is restricted to two digits values comprised between 0 and 2. To help the algorithm finding the absolute minimum of the problem, the starting $\xi$ values were chosen such that $L_{a}\left(\xi_{i}\right) \approx \lambda_{i} / 5$ with $\lambda_{i}$ the wavelength in air corresponding to the targeted frequency of the FPM $i$. The absorption coefficient $\alpha$ is maximized over the frequency range $f=[1150 ; 2000] \mathrm{Hz}$ by maximizing a cost function $J=\sum_{f} \alpha(f)$. Fourteen frequencies are considered with a linear spacing. The high frequency limit was chosen so that the highest frequencies of the bandwidth are managed by a $\xi=0$ FPM. The low-frequency limit used in this work allows a good balance between the absorption plateau frequency width and the height of its ripples. The analytically optimized $\mathrm{MpS}$ has the following parameters: $\left[\left\{\xi_{i}, I F_{i}(\%)\right\}\right]=[\{1.67,3\}$, $\{1.28,10\},\{0.72,36\},\{0,36\}] i=1,2,3$ and 4 . The corresponding JCAL parameters are presented in Tab. I. Experimentally, for $i=1$ the optimization gave the value $I F=3 \%$. However, this value is problematic as it corresponds to a lattice constant $A D=6.7 \mathrm{~mm} \approx C / 3$. Each folded cavity of this FPM should contain a single fiber per cross section. This ML is then not anymore an homogeneous porous material and its induced losses are comparable to those of the impervious walls, neglected in the models. Then, this ML is omitted during the manufacturing, i.e., $\{1.67,3\}$ is replaced by $\{1.67,0\}$.

The absorption coefficient of the optimized material is presented in Fig. 3. The green continuous line corresponds to the analytical results and the black continuous line shows the numerical predictions. The FEM predicts $\alpha(f)>0.96 \forall f \in$ [1160; 2060] Hz i.e., 5. $6 L_{b}<\lambda<9.9 L_{b}$ and $\bar{\alpha}=0.98$. The four absorption maxima are equal to 0.99 or 1.00 corresponding to the critical coupling condition for each of the FPM. The correlation between the analytical results and the numerical predictions is very good, with a slight absorption shift ( $\delta \alpha=0.01$ ) between 1500 and $2000 \mathrm{~Hz}$ mainly due to the simplification of the geometry in the analytical model. The measurement (depicted in supp. mat.) is in agreement with the theoretical predictions but the absorption is lower than expected between 1250 and $1700 \mathrm{~Hz}(\delta \alpha=0.07)$. This frequency range corresponds to the absorption maxima of the second $(i=2)$ and third $(i=3)$ FPM. Perfect absorption occurs when the losses exactly compensate the leakages of the structure $^{12}$. If the absorption is not perfect at the frequency of a maximum of absorption, it means that the associated losses are either too large or too small. Experimental investigations have shown that associated losses of the second and third FPM are too large experimentally, probably because the losses induced by the walls are neglected in the theoretical models. Then, another $\mathrm{MpS}$ was fabricated with reduced $I F$ of such FPM, i.e., with reduced inherent losses. The new MpS presents $I F_{2}(\%)=7$ and $I F_{3}(\%)=20$. This corresponds to a relative increase of $3 \%$ and $4 \%$ of the porosity respectively. The absorption coefficient of this new $\mathrm{MpS}$ is depicted by the red crosses and $\alpha(f)>0.96 \forall f \in[1155 ; 2140] \mathrm{Hz}$, i.e., $5.3 L_{b}<\lambda<9.9 L_{b}$ and $\bar{\alpha}=0.98$ is observed. The four absorption maxima are equal to 0.99 or 1.00 and are found 
logarithmically spaced in frequency as expected from [16] to achieve the mode density leading to a constant adapted impedance. The measured absorption is then in very good correlation with the theoretical predictions and slightly higher than expected above $2140 \mathrm{~Hz}$. The optimization procedure focused on maximizing the absorption in $f \in[1150 ; 2000] \mathrm{Hz}$. Above $2000 \mathrm{~Hz}$, the absorption is not controlled and oscillates between low and high values. These high values are due to the harmonics of the FPMs. Conversely, the absorption deep between $2000 \mathrm{~Hz}$ and $3350 \mathrm{~Hz}$ comes from the absence of fundamental and of harmonic resonances in this frequency range. The absorption can be brought close to unity above $2000 \mathrm{~Hz}$ and until a very high frequency by increasing the number of resonators composing the $\mathrm{MpS}$ and tuning their resonances at frequencies for which the modal density is low in the presented configuration ${ }^{16}$. Increasing the number of elements decreases the percentage of surface of the $\mathrm{MpS}$ covered by each element and decreases the absorption peaks amplitude. This can be compensated by a porous layer added on top of the $\mathrm{MpS}$ flattening the absorption dips ${ }^{16,33,34}$.

To highlight the crucial role of the MLs, that of the optimized MpS are replaced by air. The treatment is simulated analytically in the same way as the MpS but the Stinson's model $^{35}$ is used to account for the losses inside the cavities. The air filled cavities are then simulated as four $L_{a}(\xi)$ height quadruplets of QWR of hydraulic radius $Q(\xi) / \sqrt{\pi}$. The losses of such air-filled QWR are not optimal, they do not compensate the leakages. The losses are only induced by the folded geometry and thus by $\xi$. The analytically computed absorption coefficient of the air-filled folded material is depicted by green dashed line in Fig. 3. The absorption maxima are sharp and there is no absorption plateau close to unity. Moreover it is worth noting here that a single FPM produces an absorption peak at longer wavelength than an homogeneous ML with the same $L_{b}$ at the cost of a narrower absorption frequency width (Fig. 3, blue and purple dashed lines). However, the quality factor of the absorption peak of the FPM is much smaller than that of the QWR filled with air. This is the fundamental reason that explains the broadband character of the designed $\mathrm{MpS}$ in this work.

In this work, a folded metaporous material for subwavelength and broadband perfect absorption of sound is theoretically and experimentally reported. The metaporous material consists in the assembly of several FPM possessing sub-wavelength low quality factor resonances. The geometry of each FPM is optimized in order to present perfect absorption at particular frequencies within a target frequency range. Therefore the micro-lattice of the FPM is optimized to present the perfect balance between the inherent losses and the leakage of the resonator, giving rise to the critical coupling condition and as consequence to the perfect absorption. An analytical model combining the modal decomposition and the transfer matrix method as well as a full wave numerical model are used to theoretically study the system. The analytical model is used to optimize the geometry for perfect absorption of sound at normal incidence for noise having a wavelength in air such that $5.6 L_{b}<\lambda<9.9 L_{b}$. Relatively longer wavelengths could also be perfectly absorbed at the

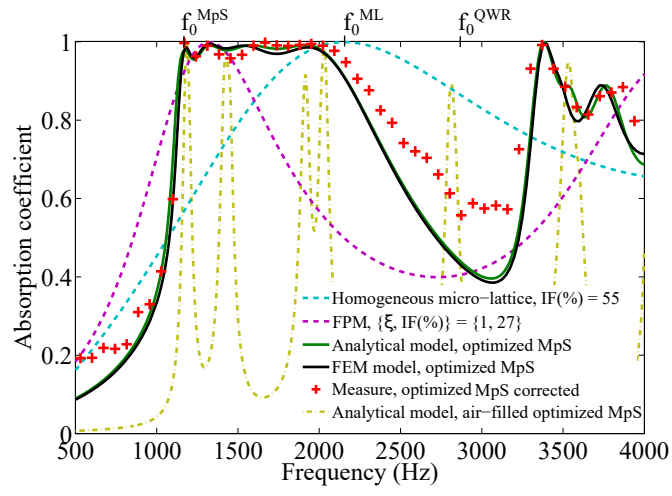

FIG. 3. (color online) Rigid-backing normal incidence absorption coefficient of $L_{b}=30 \mathrm{~mm}$ thick materials. Critically coupled: homogeneous ML (blue dashed line) and $\xi=1$ single FPM (purple dashed line). Simulation of the optimized MpS: analytical model (green solid line), FEM model (black solid line). Measurement of optimized MpS with adjusted $I F$ (red crosses). Analytical simulation of the optimized MpS replacing the MLs by air (green dashed line). The $f_{0}$ marks highlight the frequency of the first absorption maximum of a $30 \mathrm{~mm}$ thick QWR filled by air, ML and MpS.

cost of narrower bandwidth. The structure is fabricated by $3 \mathrm{D}$ printing techniques showing very good agreement with the theoretical results. Unlike folded liners, air-filled QWR or HR, the losses of the introduced treatment are governed by a porous material. In this way, the losses of the treatment are independent of its impervious folded structure.

\section{DATA AVAILABILITY}

The data that supports the findings of this study are available within the article and its supplementary material.

\section{SUPPLEMENTARY MATERIAL}

The Supplementary Material of this Letter gives further information about the equivalent fluid JCAL model and the variation of the JCAL parameters with respect to the $I F$, the manufacturing process, the numerical and analytical models including the geometry simplification of the FPMs process and finally the measurements.

\section{ACKNOWLEDGMENTS}

The authors acknowledge Safran Aircraft Engines, the Natural Sciences and Engineering Research Council of Canada (NSERC) for supporting and funding this research. They acknowledge financial support from ANR industrial 
TABLE I. JCAL parameters of the numerically optimized MpS

\begin{tabular}{|c|c|c|c|c|c|c|c|c|}
\hline$i$ & $\xi$ & $I F$ & $\phi$ & $\begin{array}{l}\alpha_{\infty}^{/ /} \\
\alpha_{\infty}^{\perp}\end{array}$ & $\begin{array}{l}\Lambda^{/ /} \\
\Lambda^{\perp} \\
(\mu \mathrm{m})\end{array}$ & $\begin{array}{l}\Lambda^{\prime} \\
(\mu \mathrm{m})\end{array}$ & $\begin{array}{l}q_{0}^{/ /} \\
q_{0}^{\perp} \\
\left(10^{-9} \mathrm{~m}^{2}\right)\end{array}$ & $\begin{array}{l}q_{0}^{\prime} \\
\left(10^{-9} \mathrm{~m}^{2}\right)\end{array}$ \\
\hline \multirow{2}{*}{1} & \multirow{2}{*}{1.67} & \multirow{2}{*}{$3 \%$} & \multirow{2}{*}{0.99} & 1.00 & 1000 & \multirow{2}{*}{3300} & 59.3 & \multirow{2}{*}{756} \\
\hline & & & & 1.04 & 2800 & & 702 & \\
\hline \multirow{2}{*}{2} & \multirow{2}{*}{1.28} & \multirow{2}{*}{$10 \%$} & \multirow{2}{*}{0.94} & 1.02 & 394 & \multirow{2}{*}{1200} & 20.1 & \multirow{2}{*}{95.2} \\
\hline & & & & 1.07 & 575 & & 95.2 & \\
\hline \multirow{2}{*}{3} & \multirow{2}{*}{0.72} & \multirow{2}{*}{$24 \%$} & \multirow{2}{*}{0.81} & 1.20 & 156 & \multirow{2}{*}{463} & 5.35 & \multirow{2}{*}{14.1} \\
\hline & & & & 1.13 & 256 & & 9.6 & \\
\hline \multirow{2}{*}{4} & \multirow{2}{*}{0} & \multirow{2}{*}{$36 \%$} & \multirow{2}{*}{0.71} & 1.35 & 100 & \multirow{2}{*}{225} & 2.20 & \multirow{2}{*}{5.5} \\
\hline & & & & 1.18 & 151 & & 2.96 & \\
\hline
\end{tabular}

chair MACIA (ANR-16-CHIN-0002).

\section{REFERENCES}

${ }^{1}$ J.-F. Allard and N. Atalla, Propagation of sound in porous media: modelling sound absorbing materials, 2nd ed. (Wiley, Hoboken, N.J, 2009).

${ }^{2}$ L. Cao, Q. Fu, Y. Si, B. Ding, and J. Yu, "Porous materials for sound absorption," Composites Communications 10, 25-35 (2018).

${ }^{3}$ Zielinski, "Pore-size effects in sound absorbing foams with periodic microstructure: modelling and experimental verification using $3 \mathrm{~d}$ printed specimens," (2016).

E. R. Fotsing, A. Dubourg, A. Ross, and J. Mardjono, "Acoustic properties of periodic micro-structures obtained by additive manufacturing," Applied Acoustics 148, 322-331 (2019).

${ }_{5}^{5}$ J. Boulvert, T. Cavalieri, J. Costa-Baptista, L. Schwan, V. Romero-García, G. Gabard, E. R. Fotsing, A. Ross, J. Mardjono, and J.-P. Groby, "Optimally graded porous material for broadband perfect absorption of sound," Journal of Applied Physics 126, 175101 (2019).

${ }^{6}$ J. Boulvert, J. Costa-Baptista, T. Cavalieri, M. Perna, E. R. Fotsing, V. Romero-García, G. Gabard, A. Ross, J. Mardjono, and J.-P. Groby, "Acoustic modeling of micro-lattices obtained by additive manufacturing," Applied Acoustics 164, 107244 (2020).

${ }^{7}$ T. Parrott, M. Jones, and B. Homeijer, "Effect of Resonator Axis Skew on Normal Incidence Impedance," in 9th AIAA/CEAS Aeroacoustics Conference and Exhibit (American Institute of Aeronautics and Astronautics, Hilton Head, South Carolina, 2003).

B. Howerton and T. Parrott, "Validation of an Acoustic Impedance Prediction Model for Skewed Resonators," in 15th AIAA/CEAS Aeroacoustics Conference (30th AIAA Aeroacoustics Conference) (American Institute of Aeronautics and Astronautics, Miami, Florida, 2009).

${ }^{9}$ X. Cai, Q. Guo, G. Hu, and J. Yang, "Ultrathin low-frequency sound absorbing panels based on coplanar spiral tubes or coplanar Helmholtz resonators," Applied Physics Letters 105, 121901 (2014).

${ }^{10}$ R. J. Beckemeyer and D. T. Sawdy, "Analytical and experimental studies of folded cavity duct acoustic liners," The Journal of the Acoustical Society of America 60, S123-S123 (1976).

${ }^{11}$ D. T. Sawdy and R. J. Beckemeyer, "Bandwidth Attenuation with a Folded Cavity Liner in a Circular Flow Duct," AIAA Journal 18, 766-773 (1980).

${ }^{12}$ V. Romero-García, G. Theocharis, O. Richoux, and V. Pagneux, "Use of complex frequency plane to design broadband and sub-wavelength absorbers," The Journal of the Acoustical Society of America 139, 3395-3403 (2016)

${ }^{13} \mathrm{~J}$.-P. Groby, R. Pommier, and Y. Aurégan, "Use of slow sound to design perfect and broadband passive sound absorbing materials," The Journal of the Acoustical Society of America 139, 1660-1671 (2016)

${ }^{14} \mathrm{Y}$. Li and B. M. Assouar, "Acoustic metasurface-based perfect absorber ${ }^{14} \mathrm{Y}$. Li and B. M. Assouar, "Acoustic metasurface-based perfect absorber
with deep subwavelength thickness," Applied Physics Letters 108, 063502 with deep subwavelength thickness," Applied
(2016), https://doi.org/10.1063/1.4941338.

${ }^{15}$ K. Donda, Y. Zhu, S.-W. Fan, L. Cao, Y. Li, and B. Assouar, "Extreme low-frequency ultrathin acoustic absorbing metasurface," Applied Physics Letters 115, 173506 (2019), https://doi.org/10.1063/1.5122704.

${ }^{16} \mathrm{M}$. Yang, S. Chen, C. Fu, and P. Sheng, "Optimal sound-absorbing structures," Materials Horizons 4, 673-680 (2017).

${ }^{17}$ L. Lagarrigue, J.-P. Groby, V. Tournat, O. Dazel, and B. Nennig, "Acoustic panel," (Patent US20160210955A1).

${ }^{18}$ J.-P. Groby, A. Duclos, O. Dazel, L. Boeckx, and W. Lauriks, "Absorption of a rigid frame porous layer with periodic circular inclusions backed by a periodic grating," The Journal of the Acoustical Society of America 129, 3035-3046 (2011), https://doi.org/10.1121/1.3561664.

${ }^{19}$ C. Lagarrigue, J. P. Groby, V. Tournat, O. Dazel, and O. Umnova, "Absorption of sound by porous layers with embedded periodic arrays of resonant inclusions," The Journal of the Acoustical Society of America 134, 46704680 (2013), https://doi.org/10.1121/1.4824843.

${ }^{20}$ J.-P. Groby, C. Lagarrigue, B. Brouard, O. Dazel, V. Tournat, and B. Nennig, "Enhancing the absorption properties of acoustic porous plates by periodically embedding helmholtz resonators," The Journal of the Acoustical Society of America 137, 273-280 (2015), https://doi.org/10.1121/1.4904534

${ }^{21}$ Y. Zhou, D. Li, Y. Li, and T. Hao, "Perfect acoustic absorption by subwavelength metaporous composite," Applied Physics Letters 115, 093503 (2019), https://doi.org/10.1063/1.5107439. 
${ }^{22}$ J.-P. Groby, W. Lauriks, and T. E. Vigran, "Total absorption peak by use of a rigid frame porous layer backed by a rigid multi-irregularities grating," The Journal of the Acoustical Society of America 127, 2865-2874 (2010), https://doi.org/10.1121/1.3337235.

${ }^{23} \mathrm{X}$. Zhu, K. Li, P. Zhang, J. Zhu, J. Zhang, C. Tian, and S. Liu, "Implementation of dispersion-free slow acoustic wave propagation and phase engineering with helical-structured metamaterials," Nature Communications 7 , 11731 (2016).

${ }^{24} \mathrm{M}$. Versaevel, L. Moreau, and E. Lacouture, "Folded spiral-shaped cavitie for nacelle acoustic liners: Impedance and attenuation modelling and comparison to experimental results," in 3AF Greener Aviation 2016 (Bruxelles, 2016) p. 9.

${ }^{25}$ D. Lafarge, P. Lemarinier, J. Allard, and V. Tarnow, "Dynamic compressibility of air in porous structures at audible frequencies," J. Acoust. Soc. Am. 102, 1995-2006 (1997).

${ }^{6}$ V. Tarnow, "Compressibility of air in fibrous materials," The Journal of the Acoustical Society of America 99, 3010-3017 (1996)

${ }^{27} \mathrm{~J}$.-L. Auriault, C. Boutin, and C. Geindreau, Homogenization of coupled phenomena in heterogenous media (2009) oCLC: 733729827.

${ }^{28}$ C.-Y. Lee, M. J. Leamy, and J. H. Nadler, "Numerical Calculation of Effective Density and Compressibility Tensors in Periodic Porous Media: A Multi-Scale Asymptotic Method," (2008).

${ }^{29}$ A. Terroir, L. Schwan, T. Cavalieri, V. Romero-García, G. Gabard, an

J.-P. Groby, "General method to retrieve all effective acoustic propertie of fully-anisotropic fluid materials in three dimensional space," Journal of
Applied Physics 125, 025114 (2019), arXiv: 1810.03603.

${ }^{30}$ M. Niskanen, J.-P. Groby, A. Duclos, O. Dazel, J. C. Le Roux, N. Poulain, T. Huttunen, and T. Lahivaara, "Deterministic and statistical characterization of rigid frame porous materials from impedance tube measurements," The Journal of the Acoustical Society of America 142, 2407-2418 (2017).

${ }^{31}$ T. Cavalieri, J. Boulvert, L. Schwan, G. Gabard, V. Romero-Garcìa, J.-P. Groby, M. Escouflaire, and J. Mardjono, "Acoustic wave propagation in effective graded fully anisotropic fluid layers," The Journal of the Acoustical Society of America 146, 3400-3408 (2019)

${ }^{32}$ W. Huang, L. Schwan, V. Romero-García, J.-M. Génevaux, and J.-P. Groby, "3D-printed sound absorbing metafluid inspired by cereal straws," Scientific Reports 9, 8496 (2019).

${ }^{33}$ S. Huang, Z. Zhou, D. Li, T. Liu, X. Wang, J. Zhu, and Y. Li, "Compact broadband acoustic sink with coherently coupled weak resonances," Science Bulletin 65, 373-379 (2020)

${ }^{34}$ J.-P. Groby, B. Brouard, O. Dazel, B. Nennig, and L. Kelders, "Enhancing rigid frame porous layer absorption with three-dimensional periodic irregularities," The Journal of the Acoustical Society of America 133, 821-831 (2013).

${ }^{35} \mathrm{M}$. R. Stinson, "The propagation of plane sound waves in narrow and wide circular tubes, and generalization to uniform tubes of arbitrary crosssectional shape," The Journal of the Acoustical Society of America 89, 550558 (1991). 


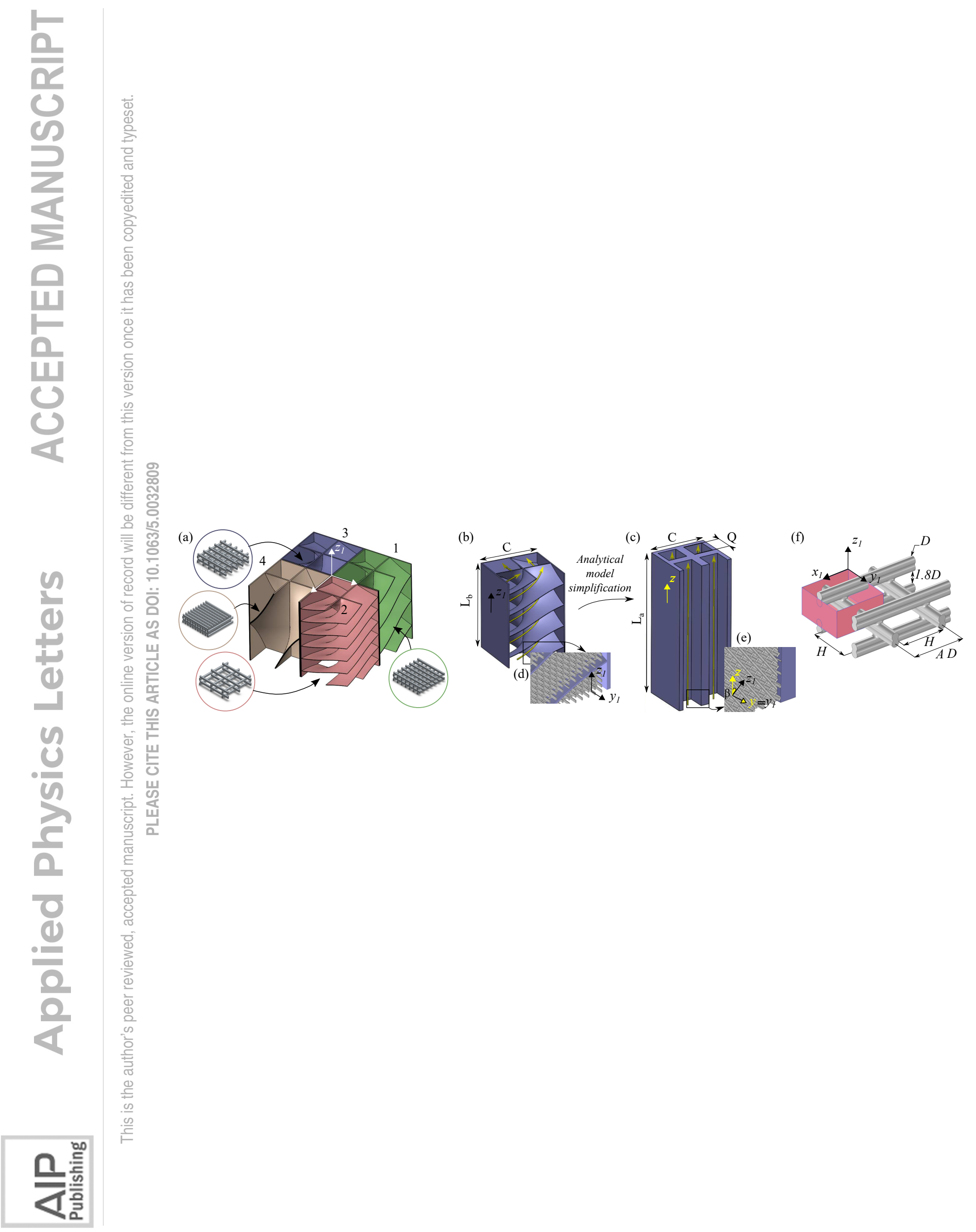




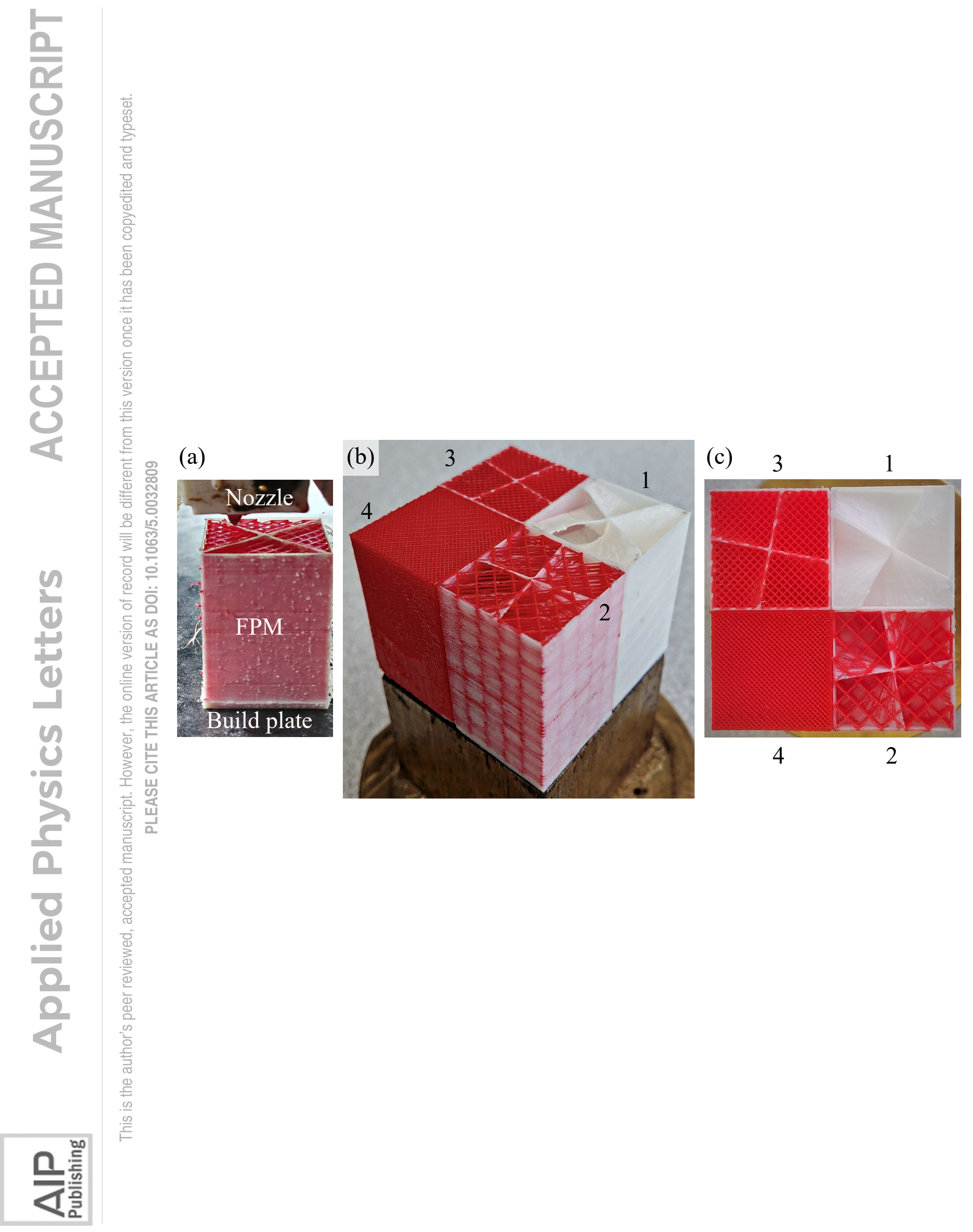




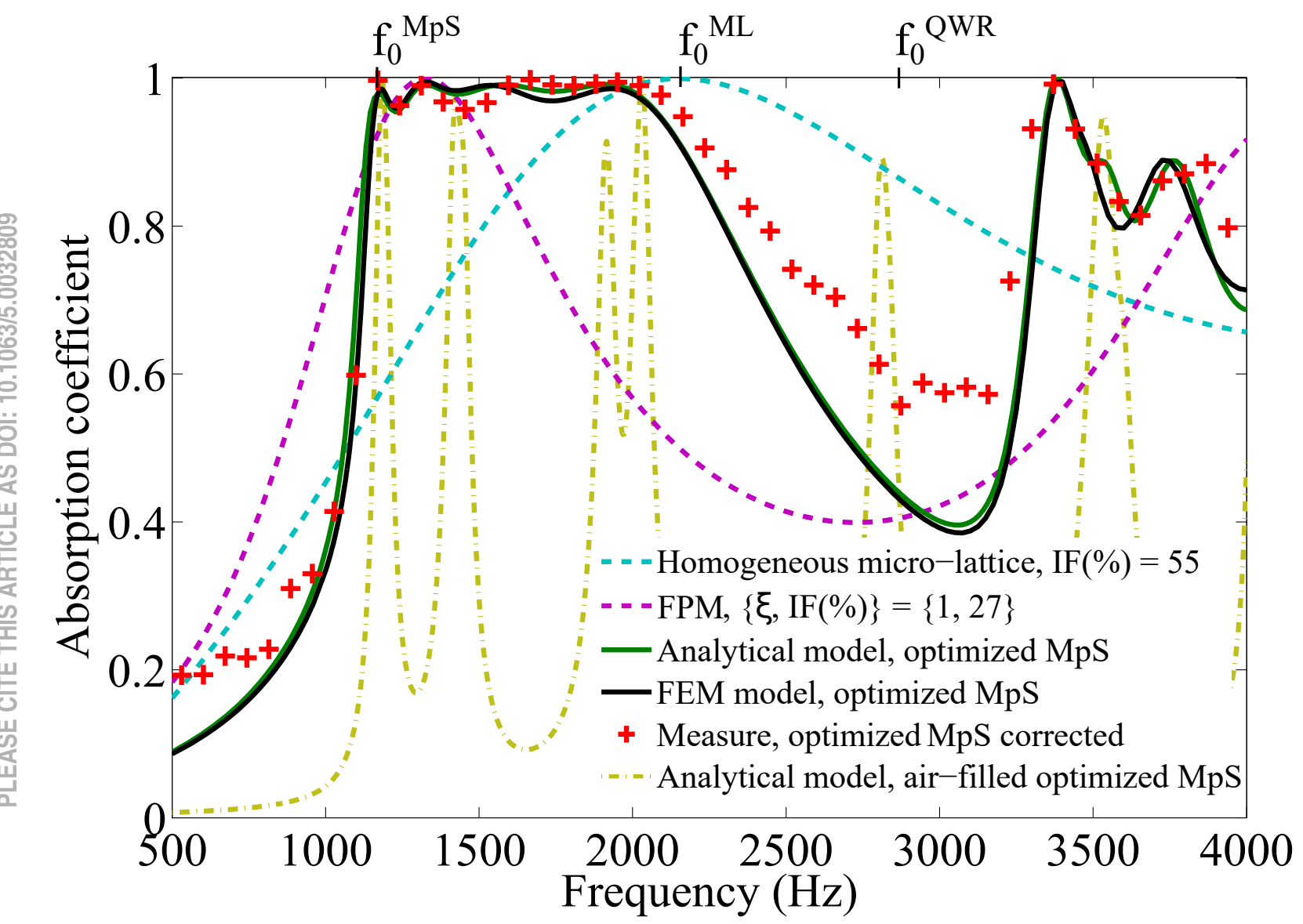

